\title{
Limits on mass of missing mass
}

\section{Of the particles whose mass may account for the missing mass of the Universe, those known as axions are the most shadowy. What is known of stellar evolution surprisingly helps to define their properties.}

IN the continuing if desultory search for the missing matter that might hold the Universe together gravitationally, the hypothetical (perhaps mythical) particle called the axion frequently crops up. The need for something along these lines follows from the evidence that clusters of galaxies appear to be more tightly bound to each other than the visible mass can account for, while the deceleration of the expansion of the Universe (poorly determined numerically) may also be more rapid than the visible mass allows. But the favourite constituent of the supposed dark matter of a few years ago, the neutrino with non-zero mass, is by no means as popular as it used to be.

For cosmologists, the attractiveness of the axion as the particle of which dark missing mass is made is much what that of neutrinos used to be. Again, the mass should be small compared with that of the electron, while the interaction of axions with other material particles should be very small, so that the chance that they would have been observed by accident in particle physics experiments would be negligible. One difficulty is that they should be produced prolifically in weak nuclear interactions, such as those responsible for beta-decay, in stellar interiors in particular. This, as it turns out, may be a difficulty. Or, more accurately, three physicists have now been able to show that what is known of the course of stellar evolution can be used to put limits on the properties of axions (see Dearborn, D.S.P., Schramm, D.N. and Steigman, G. Phys. Rev. Lett. 56, 26; 1986).

Axions (if they exist) belong to a larger family of hypothetical particles called Goldstone bosons which owe their existence (if any) to the asymmetries in weak nuclear interactions, represented for example by the non-conservation of charge and parity combined. Such doubts as there may be about the reality of the particles are congruent with those applying to the theory of the weak interaction in general, which is more and more adequately confirmed as the weeks go by.

In some respects, axions stand in relation to photons as do muons in relation to electrons: they are more massive versions of the same thing. So axions are produced by the collision of energetic photons with electrons, as in the Compton effect, or with intact atoms, when they appear as the conversion products of the original photons. They may also appear instead of photons in the collision of electrons with massive charged particles such as nucleons or nuclei, in a variant of the more familiar bremsstrahlung. But there the analogy with photons stops; once produced, axions should not interact electromagnetically with other matter but only by the weak interaction, just like neutrinos. This explains why the chances of their having been detected as if by accident are very small.

This is where the cosmology comes in. Axions produced in very large numbers in the interiors of stars and wherever else energetic photons or electrons interact with other matter will, once created, rarely be destroyed again. So the Universe will contain a sea of axions, possibly concentrated (if they have a non-zero mass) where visible matter is also concentrated, near clusters of galaxies for example. Whether their presence would account for the present estimates of the missing mass, let alone suffice to close the Universe gravitationally, is another question whose answer would depend on their number and their individual mass.

The interest in what Dearborn, Schramm and Steigman have now done is that they start from the production end of the process. If axions are copiously produced within stars, they will necessarily carry away energy and so help cool the interiors of the stars producing them. The authors do not claim that this line of argument is novel, but they do appear to have used much better models for the evolution of stars than have previously been available for this purpose.

The stage in the evolution of a star at which the influence of the axions might be critical is that of the transition of a star from the long spell (as long as $10^{10}$ years for a star like the Sun) when hydrogen is the nuclear fuel to the succeeding stage, when the hydrogen is substantially exhausted and helium becomes the next most accessible nuclear fuel. The transition is recognized as that from a main-sequence star to a red giant.

Axion production could be important at this stage because helium burning requires a higher temperature at the centre of a star. As the hydrogen is depleted, the helium produced by hydrogen burning collapses under its own weight, with the result that both the temperature and the density at the centre of the star increase, as do the radius and the luminosity of the enlarged disk. Somewhere along the line, the central temperature is enough to ignite the helium. But if the flux of axions is too great, the point may never be reached at which the helium is ignited; such an object would become redder, less luminous and less of a giant until it disappeared.

The observation that helium does ignite in stars no more massive than the Sun therefore provides a criterion for fixing an upper limit on axion production, at least with the assumption that axions once produced would travel through the envelope of a star as massive as the Sun essentially without interruption. Dearborn and his colleagues say that the fate of a star at this crucial stage will be delicately sensitive to the energy carried away by axions. Because the later stages of stellar evolution and those in which heavy elements are produced require that the transition from hydrogen to helium burning should be successfully accomplished, the character of the Universe as observed requires that there should not be too many axions.

The calculations, which entail the prediction of the evolution of a star beginning with the cosmic abundance of hydrogen and deuterium and nothing else for various assumptions about the properties of axions, are most of all a testimony to the sophistication of models of stellar evolution now in use. (The authors point out that, even so, no account is taken of the effects of rotation and of magnetic fields.) The inevitable outcome is that there is no unique upper limit for the mass of an axion, or of the other Goldstone bosons that might be substituted for it. But plausible assumptions about the stars involved and the constants that determine the production rates of axions give upper limits for the mass of an axion in the region of one electron-volt or less. Axions, if they exist at all, or other bosonic particles produced in the same way, are unlikely to carry mass greater than one millionth of the electron mass.

On the face of things, this should satisfy the cosmologists, who are looking for a particle whose interaction with other matter must be extremely feeble, which as a consequence must be long-lived and therefore abundant in the Universe, and which cannot carry more than a minute amount of mass without implying an uncomfortably large amount of missing dark matter. It will be interesting to see whether these new calculations suffice to rule out (or in) others of the notions that have recently been canvassed.

John Maddox 